\title{
Second-line triple therapy in failures with vonoprazan-based triple therapy for eradication of Helicobacter pylori
}

\author{
NAOYOSHI MORI ${ }^{1}$, YUUKI NISHIURA ${ }^{2}$, DAISUKE SUGA ${ }^{2}$, ISAO MORITANI ${ }^{2}$, YUTAKA YAMANAKA ${ }^{2}$, \\ YUMI OOYA $^{2}$, HIDEKAZU INOUE ${ }^{2}$, KOUJIROU TAKASE $^{2}$, MASATO HIOKI $^{1}$ and KATSUYA SHIRAKI ${ }^{2}$
}

Departments of ${ }^{1}$ Pharmacy and ${ }^{2}$ Gastroenterology, Mie Prefectural General Medical Center, Yokkaichi, Mie 510-8561, Japan

Received November 11, 2017; Accepted May 16, 2018

DOI: $10.3892 /$ br.2018.1111

\begin{abstract}
Gastric acid inhibition during treatment is important for the eradication of Helicobacter pylori $(H$. pylori) infection. A novel potassium-competitive acid blocker, vonoprazan (VPZ), has been demonstrated to achieve high eradication rates; however, the efficacy of second-line treatment in failures of VPZ-based triple therapy has not been well studied. The aim of the current study was to determine the efficacy of VPZ in a first-line regimen for $H$. pylori eradication, and the efficacy of a second-line regimen using metronidazole (MTZ) in failures with the first-line regimen. Of 580 subjects enrolled in the study, 524 patients completed first-line treatment (275 patients who received VPZ and 249 patients who received LPZ). First-line regimens consisted of a combination of clarithromycin (CAM) 200 or $400 \mathrm{mg}$ twice a day, amoxicillin (AMPC) $750 \mathrm{mg}$ twice a day, and either LPZ $30 \mathrm{mg}$ or VPZ $20 \mathrm{mg}$ twice a day, administered orally for 7 days. CAM and VPZ/LPZ were replaced with metronidazole (MTZ) $250 \mathrm{mg}$ and rabeprazole $10 \mathrm{mg}$ in the second-line regimens. The eradication of $H$. pylori was assessed by the $H$. pylori stool antigen test. The overall first-line eradication rate with VPZ was significantly higher than that with LPZ [91.0\% (250/275) vs. $84.7 \%$ (211/249), respectively, $\mathrm{P}=0.030]$. The dose of CAM (400 vs. $800 \mathrm{mg}$ ) did not affect the eradication rate in either the VPZ or LPZ regimens. The overall eradication rates of the second-line regimens with MTZ did not differ significantly between the VPZ-failure and LPZ-failure groups [87.0\% (20/23) vs. $87.9 \%$ (29/33), respectively, $P=0.700]$. Therefore, VPZ was significantly more effective than LPZ for first-line treatment. In patients with failure of first-line eradication therapy, successful results of second-line eradication therapy did not differ between the VPZ- and LPZ-failure groups. In
\end{abstract}

Correspondence to: Dr Katsuya Shiraki, Department of Gastroenterology, Mie Prefectural General Medical Center, 5450-132 Hinaga, Yokkaichi, Mie 510-8561, Japan

E-mail: katsuya-shiraki@mie-gmc.jp

Key words: vonoprazan, lansoprazole, Helicobacter pylori, second-line triple therapy, clarithromycin-resistant conclusion, VPZ-based triple therapy should be recommended for eradication of $H$. pylori.

\section{Introduction}

Infection with Helicobacter pylori (H. pylori) is a cause of numerous pathological conditions, including chronic gastritis, peptic ulcers, mucosa-associated lymphoid tissue lymphoma and gastric cancer $(1,2)$. Therefore, eradication of $H$. pylori is recommended for the prevention of gastric diseases. The standard therapy for $H$. pylori infection uses a proton pump inhibitor (PPI) with amoxicillin (AMPC) and clarithromycin (CAM) (3), though its success rate has decreased in multiple regions worldwide in the last 15 years. This decrease has been attributed to an increase in the prevalence of CAM-resistant strains of H. pylori. Between 2000 and 2013, a Japanese study identified that the overall resistance rate to CAM was $31.1 \%$ (2). However, in addition to bacterial resistance to antimicrobial agents, eradication failure may also be caused by insufficient acid inhibition during treatment, whereby stomach acid degrades and destabilizes the antibiotics (4). H. pylori may have increased susceptibility to antimicrobials when the microbe restores its replicative capability at a $\mathrm{pH}$ higher than 6 , since increased gastric $\mathrm{pH}$ may induce $H$. pylori to re-enter the replicative state (5). However, inhibition of gastric acid secretion by PPIs has been reported to be influenced by the cytochrome P450 (CYP) 2C19 genotype and gastric emptying (3). Therefore, stable and strong acid inhibitors are essential for the effective treatment of $H$. pylori infection.

Vonoprazan (VPZ) is a member of a novel class of gastric acid-suppressant agents, and functions as an oral potassium-competitive acid blocker by competitively inhibiting the binding of potassium ions to the $\mathrm{H}^{+}, \mathrm{K}^{+}$-ATPase in the final step of acid secretion in gastric parietal cells (3). This effect on the $\mathrm{H}^{+}, \mathrm{K}^{+}$-ATPase has been established to be potent and long lasting, afforded by high-level accumulation and slow clearance of VPZ from gastric tissues (6). Therefore, the eradication rate of $H$. pylori is expected to improve with the use of VPZ-based regimens (7).

Recently, reports have demonstrated that the acid-inhibitory effects of VPZ are more potent than those of conventional PPIs $(8,9)$. A phase III randomized trial in Japan identified that a VPZ-based triple therapy had greater efficacy as a first- or second-line triple therapy than a lansoprazole (LPZ)-based 
triple therapy (first-line eradication rate of $92.6 \%$ with VPZ vs. $75.9 \%$ with LPZ) (1). A recent multicenter study reported that VPZ-based triple therapy was effective and safe for $H$. pylori eradication in the clinic, yielding an eradication rate of up to $94.4 \%$ (9). However, in cases of failure of VPZ-based triple therapy for the eradication of $H$. pylori, second-line triple therapy has not been well studied in a clinical setting. The present study aimed to investigate the efficacy and safety of VPZ for $H$. pylori eradication as a first-line regimen, and the application of a second-line regimen using metronidazole (MTZ) in failures with a VPZ-based first-line regimen.

\section{Materials and methods}

Patients. A retrospective, open-label, single-center study design was adopted at Mie Prefectural General Medical Center (Yokkaichi, Japan). A total of 580 patients who were diagnosed with $H$. pylori infection between January 2014 and December 2016 were enrolled in the study. Among these, 308 patients had received VPZ-based triple therapy, while 272 patients had received LPZ-based triple therapy during the interval from January 2014 to December 2016. Prior to treatment, demographical and clinical characteristics including age and gender were checked. A total of 544 patients had also undergone an upper gastrointestinal endoscopy prior to enrollment. All patients diagnosed at Mie Prefectural General Medical Center $(n=450)$ received an endoscopy prior to eradication therapy as a screening process for gastric cancer. However, a total of 36 patients who were diagnosed at affiliated hospitals did not. The present study excluded patients with a history of eradication therapy and gastric operations. Informed consent was waived due to the retrospective nature of the study. The study protocol was reviewed and approved by the Ethics Committee of Mie Prefectural General Medical Center, and was conducted in accordance with the Declaration of Helsinki and ICH Guidelines for Good Clinical Practice (10).

Assessment of H. pylori infection and eradication therapy. The presence of $H$.pylori infection was detected by the rapid urease test (PyloriTek Test kit; Sekisui Medical Co., Ltd., Tokyo, Japan) or the H. pylori stool antigen HpSA test (ImmunoCard ST HpSA; Fujirebio, Inc., Tokyo, Japan).

First-line eradication regimens consisted of a combination of CAM $200 \mathrm{mg}$ twice a day or $400 \mathrm{mg}$ twice a day, AMPC $750 \mathrm{mg}$ twice a day, and either VPZ $20 \mathrm{mg}$ twice a day or LPZ $30 \mathrm{mg}$ twice a day, administered orally for 7 days. Patients were instructed to take the triple therapy once in the morning and once in the evening. Within 8 weeks after completion of the therapy, the extent of eradication of $H$. pylori infection was assessed by HpSA testing. If eradication failed (patients tested positive for HpSA), the patients underwent second-line eradication treatment. The second-line eradication regimen consisted of MTZ $250 \mathrm{mg}$ twice a day, AMPC $750 \mathrm{mg}$ twice a day and rabeprazole (RPZ) $10 \mathrm{mg}$ twice a day, administered orally for 7 days. Within 8 weeks after completion of the second-line therapy, the extent of eradication of H.pylori infection was assessed again by the feces antigen test. All patients were interviewed by a doctor to document adverse events experienced (if any) and to determine the drug compliance following completion of therapy. Adverse events were

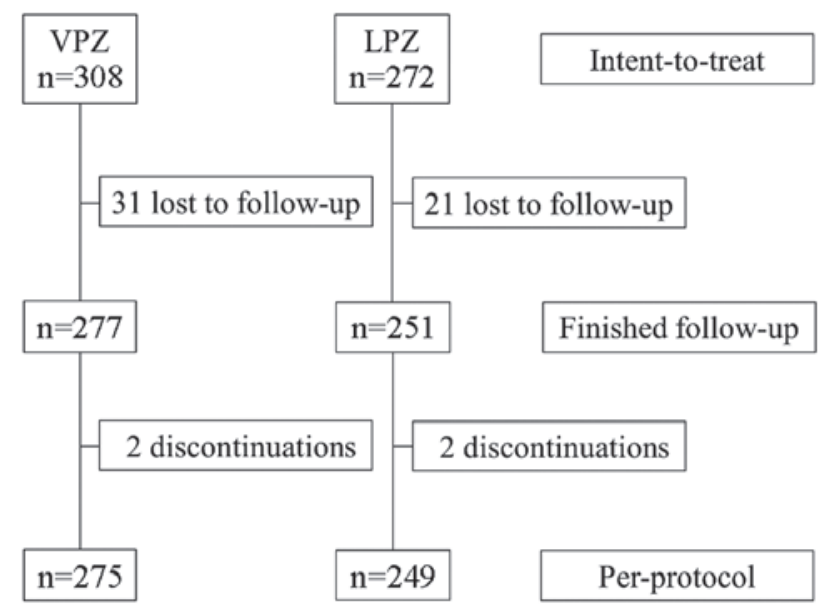

Figure 1. Flow chart of patient inclusion process. VPZ, vonoprazan; LPZ, lansoprazole.

scored using the Common Terminology Criteria for Adverse Events (CTCAE) v4.0 (11).

Data analysis. The cure rate was defined as the number of successfully treated patients divided by the number of treated patients. Data were expressed as median (range) or as n (\%) of subjects. Statistical analysis of eradication rate between the VPZ and LPZ regimens was conducted using the Fisher's exact test. Patient characteristics were compared between the VPZ and LPZ regimen groups also via Fisher's exact test. Data analysis and descriptive and inferential statistics were performed using Statcel (the useful add in forms on Excel - 2nd edition; OMS Publishing, Inc., Saitama, Japan). $\mathrm{P}<0.05$ was considered to indicate statistical significance.

\section{Results}

Characteristics of patients. A diagram of the progression of patient inclusion during the study is depicted in Fig. 1. A total of 580 subjects were included in the study (308 patients in the VPZ group; 272 patients in the LPZ group). In total, 524 patients completed the first-line treatment protocol (275 patients in the VPZ group; 249 patients in the LPZ group). There was failure of follow-up for a total of 52 patients due to personal reasons.

The baseline characteristics of each group are summarized in Table I. No significant differences were observed regarding clinical and demographic characteristics of the patients between the two treatment groups.

Comparison of first-line eradication rate. The overall first-line eradication rate was $91.0 \%(250 / 275)$ for the VPZ-based triple therapy and $84.7 \%(211 / 249)$ for the LPZ regimen. The eradication rate for VPZ-based triple therapy was significantly higher than that for LPZ-based triple therapy $(\mathrm{P}=0.030$; Fig. 2). However, there was no significant difference in the intention-to-treat eradication rate of the treatments between the two groups ( $\mathrm{P}=0.285$; data not shown), likely due to the loss at follow-up.

When comparing patient-specific eradication rates between the regimens, a significant difference was identified in patients who were male, and in those treated with the lower 
Table I. Baseline characteristics of enrolled patients in each first-line treatment group.

\begin{tabular}{lccc}
\hline Variable & VPZ/AMPC/CAM & LPZ/AMPC/CAM & P-value \\
\hline $\mathrm{n}$ & 275 & 249 & 0.572 \\
Age, years, median (range) & $62.7(27-92)$ & $61.9(26-88)$ & 0.742 \\
Sex, male:female, $\mathrm{n}$ & $154: 121$ & $143: 106$ & \\
\hline
\end{tabular}

VPZ, vonoprazan; AMPC, amoxicillin; CAM, clarithromycin; LPZ, lansoprazole.

Table II. Comparison of patient-specific first-line eradication rate.

\begin{tabular}{llll}
\hline & \multicolumn{3}{c}{ Eradication rate \% $(\mathrm{n})$} \\
\cline { 2 - 3 } Variable & VPZ/AMPC/CAM & LPZ/AMPC/CAM & \\
\hline Age, years & $91.1(174 / 191)^{\mathrm{a}}$ & $84.8(145 / 171)^{\mathrm{d}}$ & 0.064 \\
$<70$ & $90.5(76 / 84)^{\mathrm{a}}$ & $84.6(66 / 78)^{\mathrm{d}}$ & \\
$\geq 70$ & & $86.0(123 / 143)^{\mathrm{e}}$ & 0.185 \\
Sex & $94.1(145 / 154)^{\mathrm{b}}$ & $83.0(88 / 106)^{\mathrm{e}}$ & \\
Male & $87.4(105 / 121)^{\mathrm{b}}$ & $84.9(152 / 179)^{\mathrm{f}}$ \\
Female & & $84.3(59 / 70)^{\mathrm{f}}$ \\
CAM, mg & $96.4(54 / 56)^{\mathrm{c}}$ & 0.429 \\
400 & $89.9(196 / 219)^{\mathrm{c}}$ & 0.022 \\
800 & & 0.239 \\
\hline
\end{tabular}

${ }^{\mathrm{a} N o}$ significant difference $(\mathrm{P}=0.868)$; ${ }^{\mathrm{b}}$ significant difference $(\mathrm{P}=0.035) ;{ }^{\mathrm{c}}$ no significant difference $(\mathrm{P}=0.107) ;{ }^{\mathrm{d}}$ no significant difference $(\mathrm{P}=0.971)$; ${ }^{e}$ no significant difference $(\mathrm{P}=0.516)$; ${ }^{\mathrm{f}}$ no significant difference $(\mathrm{P}=0.901)$. VPZ, vonoprazan; AMPC, amoxicillin; CAM, clarithromycin; LPZ, lansoprazole.

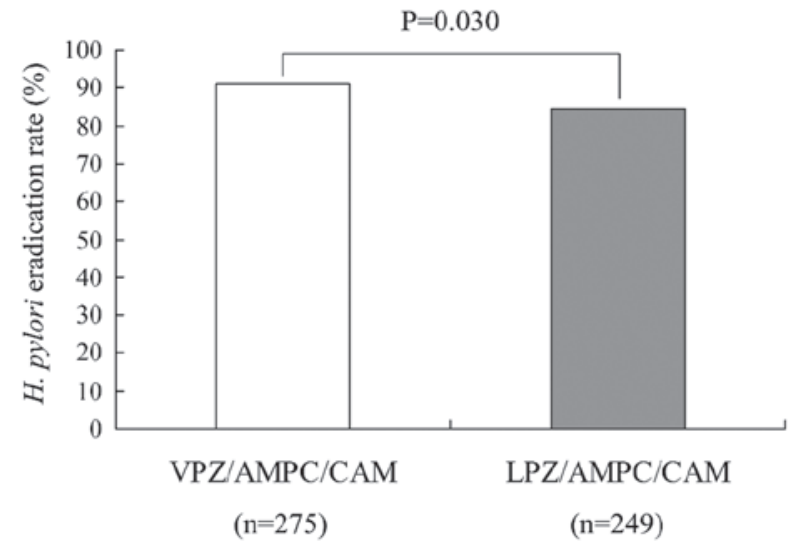

Figure 2. H. pylori eradication rate in first-line therapy with VPZ or LPZ. VPZ, vonoprazan; AMPC, amoxicillin; CAM, clarithromycin; LPZ, lansoprazole; H. pylori, Helicobacter pylori.

dose (400 mg) of CAM (Table II). Specifically, the eradication rate of the VPZ-based triple therapy was significantly higher than that of the LPZ-based triple therapy when assessed in males [94.1\% (145/154) vs. 86.0\% (123/143), respectively, $\mathrm{P}=0.018]$ and in patients dosed at $400 \mathrm{mg} \mathrm{CAM}[96.4 \%(54 / 56)$ vs. $84.9 \%$ (152/179), respectively, $\mathrm{P}=0.022]$. The dose of CAM (400 vs. $800 \mathrm{mg}$ ) did not affect the eradication rate in either the VPZ or LPZ groups. In VPZ-based triple therapy, the eradication rate in male patients was higher than that in female patients $(\mathrm{P}=0.035$; Table II $)$.

Adverse events. The VPZ and LPZ regimens were well tolerated by the patients. No severe adverse effects were reported among those who completed the protocol; however, some patients experienced less-severe adverse effects, including fever, nausea, stomach ache and rash. A total of 4 patients (2 receiving VPZ, 2 receiving $L P Z$ ) did not complete the protocol due to adverse effects (Fig. 1); these were nausea ( $n=2$ receiving VPZ), fever $(n=1$ receiving $L P Z)$ and $\operatorname{rash}(n=1$ receiving $L P Z)$.

Comparison of second-line eradication rate. The overall second-line eradication rates with use of MTZ instead of CAM were $87.0 \%$ (20/23) and $87.9 \%$ (29/33) for the VPZ-failure and LPZ-failure groups, respectively; a difference that did not achieve statistical significance (Fig. 3). There were no recurrent cases of $H$. pylori infection.

\section{Discussion}

The present study demonstrated that the first-line $H$. pylori eradication rate of VPZ-based triple therapy was significantly higher than that of LPZ-based triple therapy.

A recent phase III, randomized, double-blind study demonstrated that VPZ was non-inferior to the PPI LPZ (H. pylori 


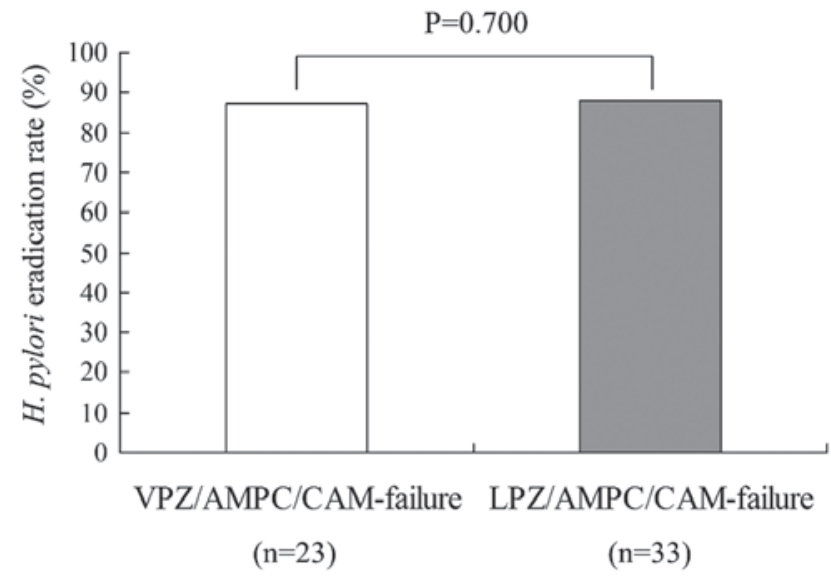

Figure 3. H. pylori eradication rate in second-line therapy following VPZfailure or LPZ-failure. VPZ, vonoprazan; AMPC, amoxicillin; CAM, clarithromycin; LPZ, lansoprazole; H. pylori, Helicobacter pylori.

eradication rate: VPZ, $92.6 \%$; LPZ, $75.9 \%$ ) when used as a component of first-line triple therapy (1). A meta-analysis incorporating 10 studies demonstrated that crude $H$. pylori eradication rates, determined by intention-to-treat analysis, were 87.9 and $72.8 \%$ with VPZ-based triple therapy and PPI-based triple therapy, respectively. The eradication rate of the VPZ-based triple therapy was superior to that of the PPI-based triple therapy [pooled risk ratio (95\% confidence interval $)=1.19(1.15-1.24)](8)$. These previous results are consistent with those of the current study.

With respect to H.pylori eradication therapy, both CAM and AMPC are acid-sensitive; therefore, inhibition of gastric acid secretions must be performed to prevent degradation of the drugs during therapy. Increased stability and bioavailability of these acid-sensitive antibiotics has been reported on inhibition of gastric acid secretion to generate a stomach $\mathrm{pH}>5(12,13)$. Furthermore, $H$. pylori may be induced by increased gastric $\mathrm{pH}$ to re-enter a replicative state, making it susceptible to antibiotics (5). Therefore, prompt, potent and long-lasting acid suppression may be critical for $H$. pylori eradication.

VPZ is a novel oral potassium-competitive acid blocker and is being increasingly used for the treatment of ulcers and reflux esophagitis in Japan $(14,15)$. Compared with LPZ, VPZ exhibits more potent and sustained acid-inhibitory effects, and induction of greater increases in gastric $\mathrm{pH}$, consistent with the observation that VPZ accumulates to higher concentrations and is cleared at a slower rate from gastric glands $(16,17)$. In addition, the intragastric $\mathrm{pH}$ following VPZ treatment ( $\mathrm{pH}$ 5.2) has been identified to be significantly higher than that following esomeprazole treatment (pH 3.0) on day 1 of therapy (18). The superior efficacy of VPZ compared with PPIs has been reported in various gastrointestinal diseases (19-22).

In the current study, the eradication rate in male patients was higher than that in female patients with VPZ-based triple therapy. In healthy Japanese subjects, a study conducted from the 1970s to the 1990s demonstrated that maximal gastric acid output gradually decreased with age in male patients while remaining constant in female patients (23). This distinction may be a reason for the higher eradication rate observed in male patients following VPZ-based triple therapy.
A significant difference in eradication rate was observed in patients treated with the lower dose of CAM $(400 \mathrm{mg})$ when comparing between the two first-line drug regimens (VPZ vs. LPZ) in the present study; this difference was not observed in patients treated with the higher dose of CAM $(800 \mathrm{mg})$. A previous report indicated that treatment with a higher dose of CAM $(800 \mathrm{mg})$ resulted in a higher eradication rate in triple therapy using conventional PPIs (24). The observation of efficacy at the lower dose in the present study presumably reflects the general efficacy of the VPZ-based triple therapy, and not superior efficacy of the lower CAM dose. Further study will be necessary to conclude whether the lower dose of CAM is superior when used as part of a VPZ-based triple therapy. Nonetheless the present results, taken together with previous reports, indicate that the VPZ-based triple therapy is more effective than a PPI-based therapy for resolving $H$. pylori infection.

However, certain patients in the present study and in previous work have experienced treatment failure with the VPZ-based triple therapy. Notably, a specific $H$. pylori mutation leading to CAM resistance appears to be primarily associated with a reduced likelihood of eradication (25). For CAM-sensitive and CAM-resistant H. pylori, the respective success rates of VPZ-based triple therapy have been reported as $97.6-100.0 \%$ and $82.0-87.5 \%$ (25). Therefore, the current study employed a combination of AMPC, MTZ and RPZ for second-line treatment. To date, there have been few reports concerning the use of second-line treatments following failures with VPZ-based first-line therapy.

The present study demonstrated that this second-line therapy achieved a high eradication rate, and that there was no significant difference in eradication rate between patients who had failed on first-line treatment with VPZ-based triple therapy or LPZ-based triple therapy. Thus, the data indicated that failure of first-line VPZ-based eradication therapy did not affect the outcome of a standard second-line eradication therapy. It may be assumed that the efficacy of second-line treatment reflects the low proportion of $H$. pylori resistant to MTZ (2-5\%) in Japan (26). Thus, acid suppression may not be a major factor of concern that influences the rate of eradication by the second-line regimen. Although certain small-scale studies have demonstrated high eradication rates with VPZ-based second-line therapy $(27,28)$, more detailed studies comparing PPIs and VPZ will be needed to clarify the optimum second-line treatment for PPI-failure or VPZ-failure patients (9).

Given that VPZ exerts a potent acid inhibitory effect, the total cost of antimicrobial drugs may be reduced by use of VPZ (3). A sub-analysis in a randomized controlled trial of VPZ indicated that the eradication rate between 400 and $800 \mathrm{mg}$ regimens of CAM did not differ significantly when using VPZ (18). In addition to the financial incentive, reductions in the treatment time and amount of antimicrobial agent used may result in a decrease in side effects when using CAM $400 \mathrm{mg}$ (3).

The current study had a number of limitations. The study followed an open-label, retrospective, single-center design, and was not systematic; therefore, the effect of CYP2C19 genotype and antibiotic resistance was not evaluated. However, regarding this point, changes in tolerance to antibiotics would have been 
presumably relatively small, since the study was performed in a single hospital over a period of 3 years, similar to previous study (3); and the sample size of the study was small, meaning further larger scale study will be needed to confirm the results.

In conclusion, the first-line $H$. pylori eradication rate of the VPZ-based triple therapy was satisfactory and significantly higher than that of the LPZ-based triple therapy among the total patient cohort, irrespective of patient background. Failure of the primary eradication with the VPZ-based first-line regimen did not affect the outcome of the second-line (MTZ-based) eradication regimen. Therefore, triple therapy with the acid blocker VPZ should be recommended for eradication of $H$. pylori.

\section{Acknowledgements}

Not applicable.

\section{Funding}

No funding was received.

\section{Availability of data and materials}

All data generated or analyzed during this study are included in this published article.

\section{Authors' contributions}

NM and KS designed and directed the project. YN, DS, IM, YY, YO and HI performed the clinical therapy and analyzed the data. KT and $\mathrm{MH}$ interpreted the data and confirmed the findings of this work. NM and KS wrote the article.

\section{Ethics approval and consent to participate}

This retrospective study was approved by the Ethics Committee of Mie Prefectural General Medical Center (Yokkaichi, Japan). Informed consent was waived due to the retrospective nature of the study.

\section{Consent for publication}

Not applicable.

\section{Competing interests}

The authors declare that they have no competing interests.

\section{References}

1. Murakami K, Sakurai Y, Shiino M, Funao N, Nishimura A and Asaka M: Vonoprazan, a novel potassium-competitive acid blocker, as a component of first-line and second-line triple therapy for Helicobacter pylori eradication: A phase III, randomised, double-blind study. Gut 65: 1439-1446, 2016.

2. McColl KE: Clinical practice. Helicobacter pylori infection. N Engl J Med 362: 1597-1604, 2010.

3. Tsujimae M, Yamashita H, Hashimura H, Kano C, Shimoyama K, Kanamori A, Matsumoto K, Koizumi A, Momose K, Eguchi T, et al: A comparative study of a new class of gastric acid suppressant agent named vonoprazan versus esomeprazole for the eradication of Helicobacter pylori. Digestion 94: 240-246, 2016.
4. Okamura T, Suga T, Nagaya T, Arakura N, Matsumoto T, Nakayama Y and Tanaka E: Antimicrobial resistance and characteristics of eradication therapy of Helicobacter pylori in Japan: A multi-generational comparison. Helicobacter 19: 214-220, 2014.

5. Graham DY and Shiotani A: New concepts of resistance in the treatment of Helicobacter pylori infections. Nat Clin Pract Gastroenterol Hepatol 5: 321-331, 2008.

6. Hunt RH: pH and Hp--gastric acid secretion and Helicobacter pylori: Implications for ulcer healing and eradication of the organism. Am J Gastroenterol 88: 481-483, 1993.

7. Sakurai Y, Shiino M, Okamoto H, Nishimura A, Nakamura K and Hasegawa S: Pharmacokinetics and safety of triple therapy with vonoprazan, amoxicillin, and clarithromycin or metronidazole: A phase 1, open-label, randomized, crossover study. Adv Ther 33: 1519-1535, 2016.

8. Jung YS, Kim EH and Park CH: Systematic review with metaanalysis: The efficacy of vonoprazan-based triple therapy on Helicobacter pylori eradication. Aliment Pharmacol Ther 46: 106-114, 2017.

9. Tanabe H, Ando K, Sato K, Ito T, Goto M, Sato T, Fujinaga A, Kawamoto T, Utsumi T, Yanagawa N, et al: Efficacy of vonoprazan-based triple therapy for Helicobacter pylori eradication: A multicenter study and a review of the literature. Dig Dis Sci 64: 3069-3076, 2017.

10. International Conference on Harmonisation of Technical Requirements for Pharmaceuticals for Human Use: ICH Harmonised Tripartite Guideline: Guideline for good clinical Practice E6 (R1). https://www.ich.org/fileadmin/Public Web Site/ICH_Products/Guidelines/Efficacy/E6/E6_R1_Guideline.pdf.

11. National Cancer Institute, National Institutes of Health, US Department of Health and Human Services: Common Terminology criteria for adverse events (CTCAE). Version 4.0.

12. Grayson ML, Eliopoulos GM, Ferraro MJ and Moellering RC Jr: Effect of varying $\mathrm{pH}$ on the susceptibility of Campylobacter pylori to antimicrobial agents. Eur J Clin Microbiol Infect Dis 8: 888-889, 1989

13. Sachs G, Meyer-Rosberg K, Scott DR and Melchers K: Acid, protons and Helicobacter pylori. Yale J Biol Med 69: 301-316, 1996.

14. Shin JM, Inatomi N, Munson K, Strugatsky D, Tokhtaeva E, Vagin $\mathrm{O}$ and Sachs G: Characterization of a novel potassiumcompetitive acid blocker of the gastric H,K-ATPase, 1-[5-(2-Fluorophenyl)-1-(pyridin-3-ylsulfonyl)-1H-pyrrol-3-yl]-Nmethylmethanamine monofumarate (TAK-438). J Pharmacol Exp Ther 339: 412-420, 2011.

15. HoriY,Imanishi A,MatsukawaJ,TsukimiY,NishidaH,ArikawaY, Hirase K, Kajino M and Inatomi N: 1-[5-(2-Fluorophenyl)1-(pyridin-3-ylsulfonyl)-1H-pyrrol-3-yl]-N-methylmethanamine monofumarate (TAK-438), a novel and potent potassiumcompetitive acid blocker for the treatment of acid-related diseases. J Pharmacol Exp Ther 335: 231-238, 2010.

16. Matsukawa J, Hori Y, Nishida H, Kajino M and Inatomi N: A comparative study on the modes of action of TAK-438, a novel potassium-competitive acid blocker, and lansoprazole in primary cultured rabbit gastric glands. Biochem Pharmacol 81: 1145-1151, 2011.

17. Hori Y, Matsukawa J, Takeuchi T, Nishida H, Kajino M and Inatomi $\mathrm{N}$ : A study comparing the antisecretory effect of TAK-438, a novel potassium-competitive acid blocker, with lansoprazole in animals. J Pharmacol Exp Ther 337: 797-804, 2011.

18. Sakurai Y, Mori Y, Okamoto H, Nishimura A, Komura E, Araki T and Shiramoto M: Acid-inhibitory effects of vonoprazan $20 \mathrm{mg}$ compared with esomeprazole $20 \mathrm{mg}$ or rabeprazole $10 \mathrm{mg}$ in healthy adult male subjects--a randomised open-label crossover study. Aliment Pharmacol Ther 42: 719-730, 2015.

19. Ashida K, Sakurai Y, Nishimura A, Kudou K, Hiramatsu N, Umegaki E, Iwakiri K and Chiba T: Randomised clinical trial: A dose-ranging study of vonoprazan, a novel potassium-competitive acid blocker, vs. lansoprazole for the treatment of erosive oesophagitis. Aliment Pharmacol Ther 42: 685-695, 2015.

20. Ashida K, Sakurai Y, Hori T, Kudou K, Nishimura A, Hiramatsu N, Umegaki E and Iwakiri K: Randomised clinical trial: Vonoprazan, a novel potassium-competitive acid blocker, vs. lansoprazole for the healing of erosive oesophagitis. Aliment Pharmacol Ther 43: 240-251, 2016.

21. Miwa H, Uedo N, Watari J, Mori Y, Sakurai Y, Takanami Y, Nishimura A, Tatsumi T and Sakaki N: Randomised clinical trial: Efficacy and safety of vonoprazan vs. lansoprazole in patients with gastric or duodenal ulcers - results from two phase 3 , non-inferiority randomised controlled trials. Aliment Pharmacol Ther 45: 240-252, 2017. 
22. Kagawa T, Iwamuro M, Ishikawa S, Ishida M, Kuraoka S, Sasaki K, Sakakihara I, Izumikawa K, Yamamoto K, Takahashi S, et al: Vonoprazan prevents bleeding from endoscopic submucosal dissection-induced gastric ulcers. Aliment Pharmacol Ther 44: 583-591, 2016.

23. Ishimura N, Owada Y, Aimi M, Oshima T, Kamada T, Inoue K, Mikami H, Takeuchi T, Miwa H, Higuchi K, et al: No increase in gastric acid secretion in healthy Japanese over the past two decades. J Gastroenterol 50: 844-852, 2015.

24. Ishioka H, Mizuno M, Take S, Ishiki K, Nagahara Y, Yoshida T, Okada H, Yokota K and Oguma K: A better cure rate with $800 \mathrm{mg}$ than with $400 \mathrm{mg}$ clarithromycin regimens in one-week triple therapy for Helicobacter pylori infection in cigarette-smoking peptic ulcer patients. Digestion 75: 63-68, 2007.

25. De Francesco V, Margiotta M, Zullo A, Hassan C, Troiani L, Burattini O, Stella F, Di Leo A, Russo F, Marangi S, et al: Clarithromycin-resistant genotypes and eradication of Helicobacter pylori. Ann Intern Med 144: 94-100, 2006.
26. Kato M, Yamaoka Y, Kim JJ, Reddy R, Asaka M, Kashima K, Osato MS, El-Zaatari FA, Graham DY and Kwon DH: Regional differences in metronidazole resistance and increasing clarithromycin resistance among Helicobacter pylori isolates from Japan. Antimicrob Agents Chemother 44: 2214-2216, 2000.

27. Katayama Y, Toyoda K, Kusano Y, Suda T, Adachi S, Terauchi I, Oka S, Takahashi M, Tamano M. Efficacy of vonoprazan-based second-line Helicobacter pylori eradication therapy in patients for whom vonoprazan-based first-line treatment failed. Gut 66: 752-753, 2017.

28. Sakurai K, Suda H, Ido Y, Takeichi T, Okuda A, Hasuda K and Hattori M: Comparative study: Vonoprazan and proton pump inhibitors in Helicobacter pylori eradication therapy. World J Gastroenterol 23: 668-675, 2017. 